Muro de la Investigación, 2019(2), agosto-diciembre ISSN: 2523-2886

DOI: https://doi.org/10.17162/r-muro-

investigaion.v4i2.843

\title{
RASGOS DE PERSONALIDAD Y CONDUCTA SEXUAL DE RIESGO EN ADOLESCENTES
}

\author{
Personality traits and risky sexual behavior in adolescents
}

\author{
Cuenca Vilchez Lizbeth Marcela ${ }^{1 \mathrm{a}}$, Lopez Ruiz Estefany Priscila ${ }^{2}$ \\ Universidad Peruana Unión, Perú ${ }^{12}$
}

Recibido: 12 de febrero de 2019

Aceptado: 22 de julio de 2019

\section{Resumen}

La presente investigación tiene como objetivo determinar si existe relación significativa entre las variables de rasgos de personalidad y conducta sexual de riesgo en estudiantes de $3^{\circ}$ a $5^{\circ}$ grado del nivel secundario de dos instituciones educativas de San Juan de Lurigancho. El estudio es de diseño no experimental, de enfoque cuantitativo, de alcance correlacional y de corte transversal. Las escalas utilizadas para la medición de las variables fueron: el cuestionario de personalidad Big Five, elaborado por Caprara, Barbaranelli y Borgogni (1993), que contiene cinco dimensiones: energía, tesón, afabilidad, estabilidad emocional y apertura mental; también se usó el cuestionario de conducta sexual de riesgo de Apaza, Estelo y Quiroz (2017). La muestra estuvo conformada por 300 estudiantes de ambos sexos entre las edades de 14 a 18 años, pertenecientes a dos instituciones educativas del distrito de San Juan de Lurigancho. Se encontró que existe relación significativa entre la dimensión "tesón" con dos conductas sexuales de riesgo: relaciones sexuales con personas desconocidas $\left(X^{2}=4.774 ; p=.029\right)$ y relaciones sexuales en fiestas con amigos $\left(\mathrm{X}^{2}=6,563 ; \mathrm{p}=.038\right)$; por su parte, la dimensión "estabilidad emocional" se relaciona significativamente con el uso del preservativo en la última relación sexual $\left(\mathrm{X}^{2}=7.491 ; \mathrm{p}=.024\right)$. Este trabajo concluye afirmando que no todos los rasgos de personalidad se relacionan con las conductas sexuales de riesgo, ya que intervienen distintos factores para que dichas conductas estén presentes en el adolescente.

Palabras claves: rasgos de personalidad, energía, afabilidad, tesón, estabilidad emocional, apertura mental, conducta sexual de riesgo, adolescencia.

\begin{abstract}
The aim of this research is to determine if there is a significant relationship between the variables of personality traits and sexual risk behavior in students from 3rd to 5th grade of the secondary level of two educational institutions of San Juan de Lurigancho. The study is a non-experimental design, quantitative approach, correlational scope and crosssectional. The scales used to measure the variables were: the Big Five personality questionnaire, prepared by Caprara, Barbaranelli and Borgogni (1993), which contains five dimensions: energy, determination, affability, emotional stability and mental
\end{abstract}

\footnotetext{
${ }^{\text {a }}$ Correspondencia al autor

E.mail: lizbethcuenca@upeu.edu.pe; prisicilalopez@upeu.edu.pe
} 
openness; The risk sexual behavior questionnaire of Apaza, Estelo and Quiroz (2017) was also used. The sample consisted of 300 students of both sexes between the ages of 14 and 18 , belonging to two educational institutions in the district of San Juan de Lurigancho. It was found that there is a significant relationship between the "tenacious" dimension with two risky sexual behaviors: sexual relations with unknown persons $(X 2=4,774 ; \mathrm{p}=.029)$ and sexual relations at parties with friends $(\mathrm{X} 2=6,563 ; \mathrm{p}=.038)$; on the other hand, the "emotional stability" dimension is related to the use of condoms in the last sexual relationship (X2 $=7.491 ; \mathrm{p}=.024)$. This paper concludes by stating that not all personality traits are related to risky sexual behaviors, since different factors are involved so that various behaviors are present in the adolescent.

Keywords: personality traits, energy, affability, determination, emotional stability, mental openness, risky sexual behavior, adolescence.

\section{Introducción}

En la actualidad, la incidencia de conductas sexuales de riesgo en adolescentes ha ido en aumento, tales como el inicio precoz de la actividad sexual, promiscuidad, relaciones sexuales sin el uso de método de protección, además bajo el efecto de algunas sustancias que muchas veces los adolescentes no son conscientes de las consecuencias que tienen sus acciones. Añadido a esto, los adolescentes experimentan una etapa del desarrollo con muchos cambios a nivel físico, emocional, social, cultural y familiar que son parte de sus vivencias, y que con el tiempo van formando rasgos de personalidad que serán parte de su vida (Paredes, 2017).

A nivel nacional, el Instituto Nacional de Estadísticas e Informática (INEI, 2016) realizó una investigación sobre salud sexual, en la cual se encontró que, del total de adolescentes peruanas entre las edades de 15 a 19 años, el 13,9\% ya estuvo embarazada alguna vez en su vida. Asimismo, al siguiente año se llegó a la conclusión que existe un incremento de madres adolescentes, entre las edades de 15 a 18 años $(17,1 \%)$. Por otro lado, el Ministerio de Salud Mental público en el 2017 los resultados de una investigación sobre salud sexual y reproductiva en adolescentes, donde se halló que, entre los años de 2000 a 2015, existe un incremento de adolescentes mujeres con inicio sexual antes de los 15 años de edad (5,1\% a 6,9\%). Por otro lado, el embarazo adolescente es el doble en el área rural en relación al área urbana (22,5\% y 10,6\%, respectivamente para el 2015). Respecto al distrito de San Juan de Lurigancho, Flores y Guevara (2005) realizaron un estudio, donde se reporta que los adolescentes de 16 años ya practican actividad sexual (32,5\%). Además, el 34,4\% tiene su primera relación sexual a los 15 años de edad, aduciendo que la principal razón para ello fue "por amor". Por consiguiente, el inicio de 
las relaciones sexuales a edades tempranas aumenta el riesgo de infecciones de transmisión sexual (ITS), porque al iniciar su actividad sexual a edades tempranas, generalmente no permanecen con una sola pareja, ni usan preservativo regularmente (Vidal y Hernández, 2017).

Ante esta problemática, es necesario enfocarse en la personalidad del estudiante, sabiendo que los adolescentes se encuentran en formación. No obstante, se quiere confirmar la posible relación entre los rasgos de personalidad y la conducta sexual, definiendo primero la personalidad como un sistema estructurado, con características singulares que poseen coherencia e individualidad en el comportamiento de una persona (Paredes, 2017; Cassaretto,2009). Según el modelo del Big Five, que plantea cinco rasgos predominantes que conforman la personalidad: "energía," donde las personas con nivel alto son activas, animosas, enérgicas y optimistas; "afabilidad," que implica confianza, honradez, altruismo, modestia; sin embargo, la persona con niveles bajos en este rasgo, es considerada egocéntrica y competitiva. El tercer rasgo es "tesón," que se caracteriza por ser competente, ordenado, obediente, autodisciplinado y responsable, pero puntajes bajos se definen como ser descuidados en sus metas y logros, tener dificultad con las normas, y un alto interés por el sexo. En base al cuarto rasgo, "estabilidad emocional," las personas con puntajes altos suelen ser tranquilas, apacibles, satisfechas consigo misma, pero, en puntajes bajos tienden a ser inquietos, temperamentales, vulnerables e irritables. Finalmente, “apertura mental," se caracterizan por sentimientos, acciones, ideas y valores presentes en el ser humano. Esta dimensión nos permite la adaptación a diferentes culturas o experiencia en el ser humano (Cassareto, 2009; Quispe, 2016; Mosquera, 2013; Sánchez y Robles, 2013).

En suma, el objetivo de este estudio es determinar si existe relación significativa entre los rasgos de personalidad y la conducta sexual de riesgo en estudiantes de $3^{\circ}$ a $5^{\circ}$ del nivel secundario en dos instituciones educativas públicas del distrito de San Juan de Lurigancho.

\section{Materiales y Métodos}

A fin de llevar a cabo esta investigación, se utilizó un diseño no experimental de corte transversal y de tipo correlacional, con la naturaleza de describir la relación entre estas dos variables: "rasgos de personalidad” y "conducta sexual de riesgo" (Hernández, Fernández y Batista, 2014). 


\section{Participantes}

Participaron 300 alumnos de dos instituciones educativas públicas de San Juan de Lurigancho que cursan el $3^{\circ}$ y $5^{\circ}$ del nivel secundario, seleccionados a través de un muestreo no probabilístico. Se incluyó a adolescente de ambos sexos, entre las edades de 14 a 18 años, los cuales completaron las encuestas.

Instrumentos

Los componentes de los cuestionarios incluyeron un documento de consentimiento informado, el cuestionario de conducta sexual de riesgo (datos sociodemográficos e información sobre conductas sexuales de riesgo) y el cuestionario de rasgos de personalidad (Big Five).

\section{Cuestionario de conducta sexual de riesgo}

El cuestionario de conducta sexual de riesgo está compuesto por 18 ítems con escala de respuestas dicotómicas (si- no) y de escala Likert (a, b, c, d, e, g, h, i, j). Fue creada partir de las investigaciones de Tarazona (2005) y de Trujillo (2015); que a su vez fueron empleadas y aplicadas por Apaza, Estelo y Quiroz (2017) en una investigación titulada "Estilos de afrontamiento y conducta sexual de riesgo en adolescentes de tres Instituciones Educativas del Distrito de Lurigancho en adolescentes de 12 a 17 años, en una población de 300 estudiantes."

\section{Cuestionario de personalidad Big Five}

Para este estudio se empleó el cuestionario Big Five (BFQ) cuyos autores originales fueron Caprara, Barbanelli y L. Borgogni (1993). Está conformado por cinco dimensiones, las cuales contienen dos subdimensiones por cada dimensión, además de una escala de distorsión. Cada sub dimensión consta de 12 elementos, (6 positivos y 6 negativos), en total suman 132 elementos. A su vez, se emplea una escala tipo Likert con opciones que van desde "completamente falso para mí" hasta "completamente verdadero para mí” y con una puntuación de 1 a 5 puntos respectivamente. La administración es individual o colectiva, y puede ser resuelto de 25 a 30 minutos. Además, este cuestionario fue adaptado y validado a la versión española por Bermúdez (1995) en su investigación en la ciudad de Madrid en una muestra de 1298 españoles 
Para el estudio también se utilizó la versión adaptada de Quispe (2016) quien realizó un estudio sobre las propiedades Psicométricas del cuestionario del Big Five de personalidad en estudiantes de institutos superiores de Huamachuco. Realizó dicho estudio en una muestra piloto de 369 estudiantes entre las edades de 16 a 55 años de institutos superiores. Se obtuvo una confiabilidad según la consistencia interna de Alfa de Cronbach por dimensiones; en energía (.75), afabilidad (.073), tesón (.079), estabilidad emocional (.087) y apertura mental (.76). Además, el estudio evaluó la validez de constructo utilizando un análisis factorial confirmatorio, donde el modelo presento aceptables niveles de adecuación

Análisis de datos

Primero, se analizó y se procesaron los resultados en el software estadístico SPSS v. 22. Además, se realizó la limpieza de los datos, y los resultados que se obtuvieron fueron ordenados en tablas de frecuencia, por lo que cada una de estas tiene una determinada interpretación. Se utilizó la prueba estadística Chi-cuadrada para determinar si existe relación significativa entre las variables de conductas sexuales de riesgo y los rasgos de la personalidad de los estudiantes encuestados de las dos instituciones.

\section{Resultados y discusión}

Dimensiones de la personalidad

En la tabla 1 se observa que el 52.3\%de los adolescentes tienen un nivel moderado en la dimensión "energía”, es decir, estos suelen mantenerse activos, establecen vínculos de comunicación con sus compañeros, y pueden expresar sus ideas y opiniones con normalidad. Por otro lado, el $77.7 \%$ de los adolescentes obtienen un nivel bajo en la dimensión "afabilidad," es decir, se caracterizan por ser pocos cooperativos y poco amigables. En otro orden de cosas, el $53.7 \%$ de los adolescentes se consideran poco reflexivos, ordenados y diligentes. Asimismo, el 49\% tienen un nivel moderado en "estabilidad emocional," es decir, los participantes se esfuerzan en mantenerse serenos, calmados, pacientes y tratan de controlar sus emociones e impulsos. Finalmente, sólo el $3 \%$ obtienen un nivel alto en "apertura mental," esto quiere decir, que pocos adolescentes se mantienen informados y mantienen interés por las cosas y experiencias nuevas. 
Tabla 1

Dimensiones de personalidad en adolescentes

\begin{tabular}{lcccccc}
\hline & \multicolumn{2}{c}{ Bajo } & \multicolumn{2}{c}{ Moderado } & \multicolumn{2}{c}{ Alto } \\
\cline { 2 - 7 } & $\mathrm{n}$ & $\%$ & $\mathrm{n}$ & $\%$ & $\mathrm{n}$ & $\%$ \\
\hline Energía & 75 & $25 \%$ & 157 & $52.3 \%$ & 68 & $22.7 \%$ \\
Afabilidad & 233 & $77.7 \%$ & 59 & $19.7 \%$ & 8 & $2.7 \%$ \\
Tesón & 161 & $53.7 \%$ & 109 & $36.3 \%$ & 30 & $10 \%$ \\
Estabilidad & 24 & $8 \%$ & 147 & $49 \%$ & 129 & $43 \%$ \\
$\begin{array}{l}\text { Emocional } \\
\text { Apertura }\end{array}$ & 222 & $74 \%$ & 69 & $23 \%$ & 9 & $3 \%$ \\
Mental & & & & & & \\
\hline
\end{tabular}

Conductas sexuales de riesgo

Se aprecia en la tabla 2, que el $28.8 \%$ de los adolescentes encuestados refieren haber iniciado ya su vida sexual. Este porcentaje indica que existe un número de encuestados que presenta una conducta sexual de riesgo, mientras que el otro $71.1 \%$ refiere no haber iniciado su vida sexual aún.

Tabla 2

Inicio de vida sexual en adolescentes

\begin{tabular}{ccc}
\hline & \multicolumn{2}{c}{ Ha tenido relaciones sexuales } \\
\cline { 2 - 3 } & $\mathrm{N}$ & $\%$ \\
\hline $\mathrm{Si}$ & 84 & $28.9 \%$ \\
$\mathrm{No}$ & 207 & $71.1 \%$ \\
\hline
\end{tabular}

En la tabla 3 se aprecia las conductas sexuales de riesgo que presentan los adolescentes, siendo el más significativo el consumo de alcohol en las relaciones sexuales con desconocidos (54.5\%). De la misma manera, existe un $32.1 \%$ de adolescentes que tuvieron relaciones sexuales con personas desconocidas. Asimismo, existe un número de adolescentes que mantienen relaciones sexuales en reuniones sociales (p. ej. fiestas) (30.5\%); la posible consecuencia de esto es la adquisición de enfermedades de trasmisión sexual, embarazos no deseados y deserción escolar. Sin embargo, existen adolescentes que hicieron uso del preservativo en la primera y última relación sexual (58.3\% y 63.2\% respectivamente). 
Tabla 3

Conducta sexual de riesgo en adolescentes

\begin{tabular}{lcccc}
\hline & \multicolumn{2}{c}{$\mathrm{Si}$} & \multicolumn{2}{c}{ No } \\
\cline { 2 - 5 } & $\mathrm{n}$ & $\%$ & $\mathrm{n}$ & $\%$ \\
\hline $\begin{array}{l}\text { Uso de preservativo en la primera } \\
\text { relación sexual }\end{array}$ & 49 & $58.3 \%$ & 35 & $41.7 \%$ \\
$\begin{array}{l}\text { Uso de preservativo en la última } \\
\text { relación sexual }\end{array}$ & 48 & $63.2 \%$ & 28 & $36.8 \%$ \\
$\begin{array}{l}\text { Consumo de alcohol en la última } \\
\text { relación sexual }\end{array}$ & 19 & $22.9 \%$ & 64 & $77.1 \%$ \\
$\begin{array}{l}\text { Consumo de droga en la última } \\
\text { relación sexual }\end{array}$ & 8 & $9.8 \%$ & 74 & $90.2 \%$ \\
$\begin{array}{l}\text { Relaciones sexuales con personas } \\
\text { desconocidas }\end{array}$ & 26 & $32.1 \%$ & 55 & $67.9 \%$ \\
$\begin{array}{l}\text { Consumo de alcohol en relaciones } \\
\text { sexuales con desconocidos }\end{array}$ & 12 & $54.5 \%$ & 10 & $45.5 \%$ \\
$\begin{array}{l}\text { Relaciones sexuales con amigos en } \\
\text { fiestas }\end{array}$ & 25 & $30.5 \%$ & 57 & $69.5 \%$ \\
$\begin{array}{l}\text { Enfermedades de Transmisión Sexual } \\
\text { Relaciones sexuales en grupo }\end{array}$ & 6 & $7.5 \%$ & 74 & $92.5 \%$ \\
$\begin{array}{l}\text { Relaciones sexuales con trabajadoras } \\
\text { sexuales }\end{array}$ & 3 & $3.9 \%$ & 73 & $96.1 \%$ \\
\hline
\end{tabular}

Análisis de correlación

Análisis entre el rasgo de personalidad energía y la conducta sexual de riesgo

En la tabla 4 se aprecia que el coeficiente Chi-cuadrado indica que el rasgo de personalidad "energía” no se encuentra asociado significativamente con ningún tipo de conducta sexual de riesgo.

Tabla 4

Asociación entre el rasgo de personalidad energía y la conducta sexual de riesgo

\begin{tabular}{lccc}
\hline & Valor & $\mathrm{gl}$ & $\mathrm{P}$ \\
\hline Uso de preservativo en la primera relación sexual & 1.009 & 2 & .604 \\
Uso de preservativo en la última relación sexual & 3.879 & 2 & .144 \\
Consumo de alcohol en la última relación sexual & .635 & 2 & .728 \\
Consumo de droga en la última relación sexual & .189 & 2 & .910 \\
Relaciones sexuales con personas desconocidas & 1.198 & 2 & .549 \\
Consumo de alcohol en relaciones sexuales con & .782 & 2 & .676 \\
desconocidos & 4.386 & 2 & .112 \\
Relaciones sexuales con amigos en fiestas & .855 & 2 & .652 \\
Relaciones sexuales en grupo & 1.453 & 2 & .483 \\
Relaciones sexuales con trabajadoras sexuales & &
\end{tabular}

Análisis entre el rasgo de personalidad afabilidad y la conducta sexual de riesgo 
Asimismo, se observa en la tabla 5 que el rasgo de personalidad "afabilidad" no se encuentra asociado significativamente con ningún tipo de conducta sexual de riesgo.

Tabla 5

Asociación entre el rasgo de personalidad afabilidad y la conducta sexual de riesgo

\begin{tabular}{llll}
\hline & Valor & $\mathrm{gl}$ & $\mathrm{P}$ \\
\hline Uso de preservativo en la primera relación sexual & 1.596 & 2 & .450 \\
Uso de preservativo en la última relación sexual & .153 & 2 & .926 \\
Consumo de alcohol en la última relación sexual & 1.387 & 2 & .500 \\
Consumo de droga en la última relación sexual & .234 & 2 & .890 \\
Relaciones sexuales con personas desconocidas & 2.865 & 2 & .239 \\
Consumo de alcohol en relaciones sexuales con & 1.721 & 1 & .190 \\
desconocidos & & & \\
Relaciones sexuales con amigos en fiestas & 1.622 & 2 & .444 \\
Relaciones sexuales en grupo & .175 & 2 & .916 \\
Relaciones sexuales con trabajadoras sexuales & .443 & 2 & .801 \\
\hline
\end{tabular}

Análisis entre el rasgo de personalidad tesón y la conducta sexual de riesgo

Referente a la tabla 6, se aprecia que existe una relación significativa entre el rasgo de personalidad "tesón" y el consumo de alcohol en relaciones sexuales con desconocidos $\left(\mathrm{X}^{2}=4.774 ; \mathrm{p}=.029\right)$. Asimismo, se evidencia que existe relación significativa entre "tesón” y el tener relaciones sexuales con amigos en fiestas $\left(X^{2}=6,563 ; p=.038\right)$. Sin embargo, no existe relación significativa con los otros ítems de conducta sexual de riesgo.

Tabla 6

Asociación entre el rasgo de personalidad tesón y la conducta sexual de riesgo

\begin{tabular}{llll}
\hline & Valor & $\mathrm{g}$ & $\mathrm{P}$ \\
\hline Uso de preservativo en la primera relación sexual & 3.041 & 2 & .219 \\
Uso de preservativo en la última relación sexual & 3.681 & 2 & .159 \\
Consumo de alcohol en la última relación sexual & 2.022 & 2 & .364 \\
Consumo de droga en la última relación sexual & 4.597 & 2 & .100 \\
Relaciones sexuales con personas desconocidas & 1.129 & 2 & .569 \\
Consumo de alcohol en relaciones sexuales con desconocidos & 4.774 & 1 & .029 \\
Relaciones sexuales con amigos en fiestas & 6.563 & 2 & .038 \\
Relaciones sexuales en grupo & .998 & 2 & .607 \\
\hline Relaciones sexuales con trabajadoras sexuales & 2.180 & 2 & .336 \\
\hline
\end{tabular}



riesgo

En cuanto a la asociación entre la relación de las conductas sexuales de riesgo y la dimensión de "estabilidad emocional," la tabla 7 muestra que en el estadístico Chicuadrado tiene asociación significativa si la última vez que tuvo relaciones sexuales usó un tipo de preservativo $\left(\mathrm{X}^{2}=7.491 ; \mathrm{p}=.024\right)$. Esto implica que, a mayor estabilidad emocional se puede ejercer control sobre las propias emociones y conductas; esto puede conducir a hacer uso de un método anticonceptivo, como el preservativo, en las relaciones sexuales.

Tabla 7

Asociación entre el rasgo de personalidad estabilidad emocional y la conducta sexual de riesgo

\begin{tabular}{|c|c|c|c|}
\hline & Valor & $\mathrm{gl}$ & $\mathrm{P}$ \\
\hline En la primera relación se usó preservativo & 1.113 & 2 & .573 \\
\hline Ultima vez que uso preservativo & 7.491 & 2 & .024 \\
\hline Tomaste alcohol & 2.651 & 2 & .266 \\
\hline Consumiste droga & 1.447 & 2 & .485 \\
\hline Relaciones con alguien que recién conociste & 2.276 & 2 & .321 \\
\hline $\begin{array}{l}\text { Consumo de alcohol en las relaciones sexuales con } \\
\text { desconocidos }\end{array}$ & .220 & 2 & .896 \\
\hline Relaciones sexuales con amigos & 1.029 & 2 & .598 \\
\hline Relaciones sexuales en grupo & .623 & 2 & .732 \\
\hline Relaciones sexuales con trabajadoras sexuales & 1.356 & 2 & .508 \\
\hline
\end{tabular}

\section{Análisis entre el rasgo de personalidad "apertura mental" y la conducta sexual de riesgo}

En cuanto a la asociación entre la conducta sexual de riesgo y la dimensión de “apertura mental," la tabla 8 muestra el estadístico Chi- Cuadrado. Éste toma un valor de (.995) en el uso del preservativo en la primera relación sexual y en la última relación sexual (.504); por otro lado, para los que consumieron alcohol en las relaciones sexuales (.948), implica que las conductas sexuales de riesgo no están asociadas significativamente con la dimensión de apertura mental.

Tabla 8

Asociación entre el rasgo de personalidad apertura mental y la conducta sexual de riesgo

\begin{tabular}{lccr} 
& Valor & gl & P \\
\hline En la primera relación usted y su pareja usaron condón & .995 & 1 & .318 \\
\hline
\end{tabular}




\begin{tabular}{lccc}
\hline Ultima vez que uso condón & .504 & 1 & .478 \\
Tomaste alcohol & .948 & 1 & .330 \\
Consumiste droga & .267 & 1 & .605 \\
Relaciones sexuales con desconocidos & 1.236 & 1 & .266 \\
Consumo de alcohol en las relaciones sexuales con & 1.721 & 1 & .190 \\
desconocidos & & & \\
Relaciones sexuales con amigos & .953 & 1 & .329 \\
Relaciones sexuales en grupo & .018 & 1 & .892 \\
Relaciones sexuales con trabajadoras sexuales & .941 & 1 & .332 \\
\hline
\end{tabular}

\section{Discusión}

La conducta sexual de riesgo en la adolescencia es una problemática de salud que ha aumentado en los últimos años, especialmente en la etapa escolar. En relación a ello, el personal administrativo de las instituciones donde se realizó el estudio, refieren que existe un alto índice de embarazos precoces, infecciones de trasmisión sexual, padres adolescentes, entre otros factores; afectando de manera significativa la calidad de vida del adolescente. Ante esta situación, se vio la necesidad de conocer la relación entre los rasgos de personalidad y la conducta sexual de riesgo.

En este estudio, se encontró que no existe relación significativa entre el rasgo de personalidad "energía" y las conductas sexuales de riesgo, lo que evidencia que en este grupo los indicadores de intraversión o extraversión presentes en los jóvenes no necesariamente se relacionan con las conductas de riesgo en los adolescentes. Asimismo, se evidencia que las conductas sexuales de riesgo se relacionan con aquellas personas que están involucradas en el consumo excesivo de alcohol y drogas, mostrando comportamientos que atentan contra su vida: agresiones, victimización y relaciones sexuales deliberadas, abuso de sustancias, entre otras. Cabe resaltar que, si la gravedad del trastorno y la sintomatología son altos, junto a la comorbilidad entre los síntomas clínicos y trastornos de personalidad, sumado a la edad de inicio del trastorno, mayores serán las conductas de riesgo (Loinaz, Echeburua \& Irureta, 2011).

En otro orden, no se encontró relación significativa entre el rasgo de personalidad "afabilidad" y la conducta sexual de riesgo ( $>0.05)$. Esto implica que las características de este rasgo (cordialidad, servicio, realizar actividades que influyan en los demás, ser compasivos, altruistas, desconfiados, irritables, poco amigables), no necesariamente se relacionan con conductas sexuales de riesgo; debido a que en nuestra población pueden influir otros factores tales como el entorno familiar (familias monoparentales, violencia intrafamiliar), económico, escolar y social (Parra y Pérez, 2010; Yalán, 2016). Este 
resultado coincide a lo encontrado por Paredes (2017) quien halló puntajes bajos en el rasgo de personalidad "afabilidad;" por lo tanto, indicó que no está relacionados con la conducta sexual de riesgo. (Quispe, 2016; Flores, 2016; Morales, et al., 2017).

En la dimensión de "tesón” se evidenció asociación significativa con dos conductas sexuales de riesgo: el consumo de alcohol en relaciones sexuales con desconocidos $\left(X^{2}=4.774 ; p=.029\right)$ y relaciones sexuales con amigos $\left(X^{2}=6,563 ; p=.038\right)$. Esto indica que los adolescentes que se caracterizan por ser desorganizados, negligentes, perezosos y poco resilientes a las dificultades presentan ese resultado. De la misma manera, los puntajes bajos indican que no poseen la capacidad de ser tenaces, ni logran cumplir con sus objetivos planteados; por lo cual estos adolescentes tienden hacia lo más fácil de realizar, y no se preocupan por el cumplimiento de sus metas (culminación de sus estudios, el inicio de alguna carrera profesional, etc.). Como consecuencia de estas características posiblemente conduzca al adolescente a practicar relaciones sexuales con personas desconocidas (Quispe, 2016; Paredes, 2017; Sánchez y Robles, 2013).

Asimismo, se encontró que la mayor incidencia de relaciones sexuales sin protección sucede por iniciativa del género masculino, aunque los varones que han tenido relaciones sexuales con el uso de un método de protección, poseen mayor nivel de responsabilidad. En el mismo orden de cosas, se halló que la mayoría de las mujeres tienen un puntaje alto de responsabilidad, teniendo como resultado una buena calidad en sus relaciones de parejas (solo una pareja sexual, con compromiso, evitar relaciones con personas desconocidas, etc.). Esto indica que, a mayor responsabilidad disminuye la probabilidad de tener relaciones sexuales sin protección y una buena calidad de relaciones de parejas (Fernández, et al. 2013; Paredes, 2017). Por otro lado, se evidencia que los adolescentes que tienden a afrontar o prevenir los problemas y a tomar decisiones adecuadas, poseen niveles altos en responsabilidad, por lo que no suelen cometer errores a diferencia de los demás casos (Cassaretto, 2009). Con ello se confirma la hipótesis de que existe relación significativa del rasgo de personalidad "tesón" y la conducta sexual de riesgo en estudiantes de $3^{\circ}$ a $5^{\circ}$ del nivel secundario en dos instituciones educativas públicas del distrito de San Juan de Lurigancho.

Igualmente, se evidenció una relación significativa entre el rasgo de personalidad "estabilidad emocional" y la conducta sexual de riesgo en base a la pregunta "si la última vez usó preservativo para tener relaciones sexuales" $\left(X^{2}=7.491 ; p=.024\right)$. Esto envuelve que, a mayor control de emociones e impulsos mayor es el uso de preservativos como método de protección sexual. Sin embargo, lo opuesto de ser una persona estable es 
mostrar una conducta temperamental, vulnerable, emotiva e impulsiva, presentando pocas estrategias para manejar situaciones que ponen en peligro la vida de los adolescentes y tener posibles consecuencias de contraer alguna infección de transmisión sexual o embarazo adolescente. Estos datos coinciden con la investigación de Paredes (2017), quien concluye que los adolescentes que presentan inestabilidad emocional o una puntuación baja en estabilidad emocional son aquellos más propensos a desarrollar conductas sexuales de riesgo. Así también, referente al uso del preservativo, Morales et al. (2017) encontraron que los niveles bajos en estabilidad emocional tienen actitudes menos favorables hacia el preservativo, es decir, menos intención de protegerse de las ITS. Por lo tanto, la persona emocionalmente estable se caracteriza por mantenerse calmada, paciente, serena y por tomar decisiones pensadas al momento de utilizar un preservativo en la relación sexual. En base al grupo evaluado, los estudiantes obtuvieron un nivel moderado en estabilidad emocional, lo cual indica que gran parte de la población se esfuerza por mantenerse serenos, calmados, pacientes, y tienden a controlar sus impulsos y emociones (Bahamón, et al., 2014; Gómez, José y Eceiza, 2016).

Por otro lado, no se encontró asociación significativa entre el rasgo de personalidad "apertura mental" y la conducta sexual de riesgo. Las características de este rasgo se ven influenciadas por factores culturales, aptitud educacional y no necesariamente se relaciona de forma directa con la conducta sexual de riesgo.

Finalmente, se encontró que solo los rasgos de personalidad como "tesón” y "estabilidad emocional" se relacionan significativamente con la presencia de conductas sexuales de riesgo en los adolescentes evaluados. Además, es alarmante los datos estadísticos encontrados, sobre el inicio de relaciones sexuales en los adolescentes, que a menor edad inician su vida sexual.

\section{Recomendaciones}

En cuanto a la investigación sobre el tema:

- Considerar la ampliación del tamaño de la muestra, con el propósito de conocer si los resultados son similares o distintos en otros distritos aledaños a la zona.

- Considerar, para las posteriores investigaciones, la posible relación con otras variables más cercanas con conducta sexual de riesgo como: habilidades sociales, estilos de crianza, etc. 
- Considerar la utilización de otros instrumentos de evaluación de la conducta sexual de riesgo, que posea determinadas dimensiones, con el objetivo de facilitar la correlación entre otras variables.

Dirigidas a las instituciones educativas, de salud y gubernamentales:

- Implementar programas de intervención en los adolescentes, enfatizando el tema de la educación sexual, informándoles sobre las consecuencias de las conductas sexuales de riesgo, como: infecciones de transmisión sexual, embarazo adolescente, etc.

- Implementar los programas de desarrollo personal poniendo énfasis en los proyectos de vida.

- Implementar y desarrollar programas de escuela para padres, enfatizando temas de salud sexual en los niños y adolescentes.

- Brindar capacitación en forma continua y sostenida a los tutores y docentes sobre la conducta sexual de riesgo con el fin de prevenir posibles consecuencias en los estudiantes.

\section{Referencias}

Bahamón, M., Vianchá, M., \& Tobos, A. (2014). Prácticas y conductas sexuales de riesgo en jóvenes: una perspectiva de género. Revista Científica de América Latina del Caribe, España y Portugal, 31(2), 327-353. Recuperado de http://www.redalyc.org/articulo.oa?id=21331836009

Bouchard, S. Godbout, N. \& Sabourin, S. (2009) Sexual Attitudes and activities in women with borderline personality disorder involved in romantic relationships. Journal of sex and marital Terapy, 35 (2), 106-121. Doi: 10.1080/00926230802712301.

Bouniot, S., Muñoz, C., Norambuena, N., Pinto, C. y Muñoz, M. (2017). Prevalencia de conductas sexuales de riesgo en estudiantes de primer año de pregrado de la Universidad San Sebastián, Concepción, Chile, 2016: estudio descriptivo. Revista Colombiana de Obstetricia y Ginecología, 68(3), 176-185. Recuperado de:http://www.redalyc.org/articulo.oa?id=195255662003.

Brooks, T., Harris, S. Thrall, J. \& Woods, E. (2002) Association of adolescent risk behaviors with mental health symptoms in high school students. Journal of 
adolescent health. 31, 240-246. Recuperado de:

https://www.ncbi.nlm.nih.gov/pubmed/12225736

Cassaretto, M. (2009). Relación entre las cinco grandes dimensiones de la personalidad y el afrontamiento en estudiantes preuniversitarios de Lima Metropolitana. (Tesis para optar Maestría con mención en Psicología Clínica). Universidad Nacional de San Marco. Perú. Recuperado de:

http://cybertesis.unmsm.edu.pe/bitstream/handle/cybertesis/619/Cassaretto bm.pdf;js

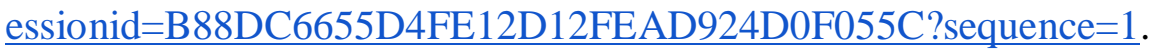

Castillo, L., Álvarez, A., Bañuelos, Y., Valle, M., Valdez, C. y Kantún, M. (2017).

Edad, género y resiliencia en la conducta sexual de riesgo para ITS en adolescentes al Sur de México. Revista electrónica trimestral de enfermería, 45, 168-175. Doi: http://dx.doi.org/10.6018/eglobal.16.1.234921.

Compte, E. (2012). Imagen corporal y conductas sexuales de riesgo en adolescentes: un estudio teórico. Revista psicológica GEPU, 3(2), 235-254. Recuperado de: http://repositorio.ub.edu.ar/bitstream/handle/123456789/4785/Psicolog\%C3\%ADa1. pdf?sequence $=1$

Fernández, A., Celis, K., Córdova, N., Dufey, M., Corréa, M., \& Benedetti, J. (2013). Sexualidad juvenil: Practicas, actitudes y diferencias según el sexo y variables de personalidad en universitarios chilenos. Revista Med chilena, 141(2), 160-166. Chile. Recuperado de: https://scielo.conicyt.cl/scielo.php?script=sci_arttext\&pid=S0034$\underline{98872013000200003}$

Flores, A. y Guevara, C. (2005). Comportamiento sexual y uso de anticonceptivos modernos en adolescentes escolares con actividad coital-San Juan de Lurigancho 2004 (Tesis de licenciatura no publicada). Universidad Mayor de San Marcos. Perú. Recuperado de:

http://cybertesis.unmsm.edu.pe/bitstream/handle/cybertesis/363/Flores_ad.pdf?seque $\underline{\text { nce }=1}$

Flores, K. (2016). Dimensiones de la personalidad y autocontrol en estudiantes del 5 to de secundaria de una institución educativa estatal, Chiclayo 2016 (Tesis de licenciatura). Universidad Señor de Sipán. Perú. Recuperado de: http://repositorio.uss.edu.pe/bitstream/handle/uss/3087/Tesis\%20Dimensiones\%20de\%20la\%20Personalidad\%20y\%20Autocontrol.pdf?sequence $=1 \&$ is Allowed $=\mathrm{y}$. 
Gómez, J., José, M. y Eceiza, A. (2016). Disposición al riesgo en la actividad sexual de adolescentes: El rol de los modelos internos de apego, de las relaciones de apego con padres e iguales y de las dificultades de regulación emocional. Revistas anales de psicología, 32 (3), pp. 900. Doi: http://dx.doi.org/10.6018/analesps.32.3.221691.

Head, S. Zweimueller, S. Marchena, C. \& Hoel, E. (2014). Women's lives and challenges: Equality and empowerment since 2000. Maryland, USA: ICF International. Recuperado de: https://www.usaid.gov/sites/default/files/documents/1864/WomensLivesandChalleng esEqualityandEmpowerment.pdf.

Hernández, R., Fernández, C., \& Baptista, P. (2014). Metodología de la investigación. (6 $6^{\circ}$ ed.). México: McGraw-Hill, S.A. Recuperado de: http://observatorio.epacartagena.gov.co/wp-content/uploads/2017/08/metodologiade-la-investigacion-sexta-edicion.compressed.pdf.

Instituto Nacional de Estadística e Informática (INEI, 2015). Las adolescentes y su comportamiento reproductivo. Recuperado de:

https://www.inei.gob.pe/media/MenuRecursivo/publicaciones digitales/Est/Lib1199/ index.html.

Instituto Nacional de Estadística e Informática (INEI, 2016). Salud infantil. Recuperado de:

https://www.inei.gob.pe/media/MenuRecursivo/publicaciones_digitales/Est/Lib1151/ pdf/cap009.pdf.

Loinaz, I., Echeburua, E. y Irureta, M. (2011) Trastornos mentales como factor de riesgo de victimización violenta. Behavioral Psychology/ Psicología conductual de la Universidad del País Vasco,19(2) 421-438. Recuperado de:

http://www.ub.edu/geav/wp-content/uploads/2017/06/Loinaz-2011.TRASTORNOSMENTALES.pdf

Ministerio de salud (2017). Situación de Salud de los adolescentes y jóvenes en el Perú. Biblioteca del Ministerio de Salud, p. 81- 87. Recuperado de http://bvs.minsa.gob.pe/local/MINSA/4143.pdf.

Molano, L., Montes, C., Moreno, D., Nova, L., Olaya, E. y Páez, J. (2017). Relación entre comportamientos de riesgo en sexualidad y familia en adolescentes escolarizados, 2015-2016. Revista carta comunitaria, 25(144), 16-22. Recuperado de:

https://revistas.juanncorpas.edu.co/index.php/cartacomunitaria/article/view/151/149. 
Morales, A., Méndez, X., Orgilés, M. \& Espada, J. (2017). Personality profiles of sexual risk among spanish adolescents. Revista de Psicología clínica con niños y adolescentes, 4(1), 41-47. Recuperado de: http://www.revistapcna.com/sites/default/files/16-21.pdf.

Mosquera, B. (2013) validación del test psicométrico de la personalidad Big Five, caso de estudio aplicado en una empresa se servicio público. (Tesis para obtención de Maestría en desarrollo del talento humano). Universidad Andina Bolívar. Ecuador

Napa, G. (2015). La sexualidad con responsabilidad y cuidado en la adolescencia en los conocimientos sobre sexualidad en adolescentes en una institución educativa 2014(Tesis para optar el título de licenciatura en enfermería no publicada). Universidad Nacional Mayor de San Marcos. Perú. Recuperado de: http://cybertesis.unmsm.edu.pe/bitstream/handle/cybertesis/4056/Napa_ag.pdf?seque nce $=1 \&$ is Allowed $=\mathrm{y}$.

Paredes, F. (2017). Prevalencia de rasgos de personalidad en conductas sexuales de riesgo en adolescentes de 14 a 18 años de edad caso: Unidad Educativa Luis A. Martínez (Tesis para optar el título de licenciatura de psicología con mención en psicología clínica no publicada). Pontificia Universidad Católica del Ecuador Sede Ambato. Ecuador. Recuperado de: http://repositorio.pucesa.edu.ec/bitstream/123456789/1891/1/76394.pdf.

Parra, J. y Pérez, R. (2010). Comportamiento sexual en adolescentes y su relación con variables biosociales. Revista Perinatología y Reproducción Humana, 24(1), 7-19. Recuperado de: http://www.medigraphic.com/pdfs/inper/ip-2010/ip101b.pdf.

Quispe, (2016) Propiedades psicométricas del cuestionario Big Five de personalidad en estudiantes de institutos superiores de Huamachuco. (Tesis para obtener el título de licenciado en psicología. Universidad Cesar Vallejo. Perú.

Rojas, R. (2011). Relación entre conocimientos sobre salud sexual y las conductas sexuales de riesgo en adolescentes de secundaria. I.E.M. Nuestra Señora de Montserrat, Lima 2010 (tesis para optar el título de licenciatura en enfermería no publicada). Universidad Nacional Mayor de San Marcos. Perú. Recuperado de: http://cybertesis.unmsm.edu.pe/bitstream/handle/cybertesis/1302/rojas lr.pdf?sequen $\underline{c e}=1$.

Sánchez, T, \& Robles, B. (2013) El modelo del Big Five de personalidad y conducta delictiva. Revista International Journal of psycological Research. 6(1), 102-109. 
Sansone, R. \& Sansone, L. (2011) Sexual Behavior in Borderline Personality: Areview.

Innovations in Clinical Neuroscience, 8(2), 14-18. Recuperado de:

https://www.ncbi.nlm.nih.gov/pubmed/21686143

Uribe, A., Castellanos, J. y Cabán, M. (2016). Conductas sexuales de riesgo y comunicación sobre sexualidad entre padres e hijos universitarios. Revista de psicología Universidad de Antioquía, 8(2), 27-48. DOI:

10.17533/udea.rpsua.v8n2a03.

Vidal, E. y Hernández, B. (2017). Conductas sexuales de riesgo asociadas a las infecciones de transmisión sexual en adolescentes de una comunidad. Revista Habanera de Ciencias Médicas, 16(4), 626-627. Recuperado de: http://www.redalyc.org/pdf/1804/180453380014.pdf.

Yalán, B. (2016). Conductas sexuales de riesgo en adolescentes del Colegio Fe y Alegria $N^{\circ} 41$ La Era-Ñaña (Tesis para optar el título de licenciatura en enfermería no publicada). Universidad Ricardo Palma. Perú. Recuperado de: http://repositorio.urp.edu.pe/handle/urp/981. 